\title{
Developing a combined Light Detecting And Ranging (LiDAR) and Building Information Modeling (BIM) approach for documentation and deformation assessment of Historical Buildings
}

\author{
Reda Yaagoubi ${ }^{1}$ and Yehia Miky ${ }^{2,3}$ \\ ${ }^{1}$ College of Geomatics and Surveying Engineering, IAV Hassan II, Rabat, Morocco. \\ ${ }^{2}$ Department of Geomatics, Faculty of Environmental Design, KAU University, Jeddah, Saudi Arabia. \\ ${ }^{3}$ Department of Civil Engineering, Faculty of Engineering, Aswan University, Egypt.
}

\begin{abstract}
Cultural heritage plays a fundamental role in preserving the collective memory of a nation. However, it is noted that many historical buildings suffer from serious deformation that may lead to deterioration or loss. In this paper, we propose an approach for documentation and deformation assessment of historical buildings based on the combination of Terrestrial Light Detecting And Ranging (LiDAR) technology and Building Information Models (BIM). In order to digitally archive the current state of a historical building, classical surveying techniques (Traversing, Levelling and GPS) are integrated with Terrestrial Laser scanner (TLS). A Leica Scan Station C10 is used to accomplish the 3D point cloud acquisition. In addition, Leica GNSS Viva GS15 receivers, a Leica Total Station TCR 1201+ and a Leica Runner 24 are used for classical surveying. The result is a $3 \mathrm{D}$ point cloud with high resolution, which is referenced according to the local geodetic reference system Ain el Abd UTM 37N. This point cloud is then used to create a 3D BIM that represents the ideal condition of the building. This BIM also contains some important architectural components of the historical building. To detect and assess the deformation of building's parts that require an urgent intervention, a comparison between the 3D point cloud and the 3D BIM is performed. To achieve this goal, the main parts of the building in the BIM model (such as ceilings and walls) are compared with the corresponding segments of the $3 \mathrm{D}$ point cloud according to the normal vectors of each part. A case study that corresponds to a historical building in Jeddah Historical City named 'Robat Banajah' is presented to illustrate the proposed approach. This building was built to serve pilgrims that want to perform the fifth pillar of Islam. Then, it was endowed (waqf) as a charity housing for widows and disabled. The results of assessing deformations of the case study show that some rooms are in a degraded condition requiring urgent restoration (distortions reach up to $22 \mathrm{~cm}$ ), while other building parts are in a non-critical condition.
\end{abstract}

\section{Introduction}

Jeddah city is located on the Red Sea coastline, in the west side of $\mathrm{Al} \mathrm{Hijaz} \mathrm{region.} \mathrm{It} \mathrm{is} \mathrm{considered} \mathrm{as}$ theeconomic and the touristic capital of the Kingdom of Saudi Arabia. Also, its locationclose to the two holy cities of Islam, Makkah and Medina, gives this place a fundamental role in the history of Islamic Civilization.

Its old city contains several historical buildings that have their own characters. These buildings take a major place in the collective memory of the Saudi society. In addition, they are considered as attractive landmarks for visitors that come from all over the world. Unfortunately, some monuments inside the old city of Jeddah are under deteriorating conditions due to various human and natural factors (climate, disasters...). Hence, it is necessary to detect and repair any potential deformation at an early stage to preserve the loss of valuable historical buildings.
To do so, monitoring and analysis of structural deformations must be performed to check the current condition of such buildings [1].

This study aims to develop an approach for documenting historical buildings and measuring their deformation by combing LiDAR (Light Detecting and Ranging) technology and BIM (Building Information Modeling). To achieve this goal, a comparison between a 3D BIM model (that represents the ideal condition of the building) and a 3D LiDAR point cloud (that represents the current condition of the building) is conducted. In the following sections, an overview of methods used for documentation and deformation assessment ispresented. Then, the proposed methodology based on the combination of BIM and terrestrial LiDAR is described. Finally, a case study that corresponds to a historical building in Jeddah Historical City named 'Robat Banajah' is presented to illustrate the proposed approach. 


\section{General overview of methods for heritage documentation and deformation assessment}

\subsection{Heritage documentation methods}

The documentation of a historical building may be defined as the process of acquiring, processing, presenting and recording the required data for the determination of the current position and form according to a specific coordinate system [2]. The selection of an appropriate method depends mainly on several factors such as: 1) the required level of details, 2) the accuracy of the acquisition method, 3) the completeness of the model to be generated and 4) the maintainability of the current conditions without any alteration due to the acquisition process.

As mentioned previously, data acquisition is the first step in the documentation process. In the following, three methods thatmay be used for acquiring data in the documentation process will be presented: 1) Total Station, 2) Close-Range Photogrammetry and 3) Terrestrial LiDAR. These three methods do not require any direct contact with the object or the building during the acquisition process.

\section{Total Station:}

A total station is a traditional surveyinginstrument that allows measuring horizontal and vertical angles of a point of interest, in addition to the slope distance to that point. Furthermore, it can also make surveying computation with angle and distance observations. Therefore, the coordinates of the measured point are calculated in real time, according to a specific coordinate system. These functionalities have made total station one of the predominant instruments used in surveying nowadays.

\section{- $\quad$ Close-Range Photogrammetry (CRP):}

Close-Range Photogrammetry (CRP) is the art, science, and technology used for obtaining precise mathematical measurements and three-dimensional (3D) data model from two or more images captured with a camera at close range[3]. It allows recording the current position, form and other architectural aspects of the historical building by photographs. If more than two photos are captured, a bundle adjustment solution is possible, including all available measurements on photos at the same time [4]. The 3D model generated from a photogrammetric process may be converted to a set of $3 \mathrm{D}$ data points with a very high density [5].

\section{- Terrestrial LiDAR:}

Terrestrial LiDAR, also called Terrestrial Laser Scanning, provides remotely sensed 3D data that capture the surrounding environment. Terrestrial LiDAR is based on the use of a continuous laser beam to measure the distance between the target and the scanner. While this scanner rotates around its vertical axis, its mirror also moves the emitted laser beam up and down to cover most of the surrounding area. In general, two main techniques are used to compute the distance to the target: pulse- based and phase-based measurements [6]. Pulse-based LiDAR convert the travelling time that takes some of the emitted energy pulses to return-back to the scanner. Then, this time is converted to distance between the scanner and the target taking into consideration that the laser pulse has a speed of light. Regarding phase-based LiDAR, the scanner measures the phase shift between the emitted and returned signal. This difference in phase is proportional to the traveling time, so it can also be converted to distance between the target and the scanner.

In addition to the distance measurement, additional data including the horizontal angle of the rotating laser and vertical angle of the oscillating mirror are recorded. The scanner automatically combines all these data to calculate an accurate $3 \mathrm{D} \mathrm{X}, \mathrm{Y}$, and $\mathrm{Z}$ coordinates position for each point. The set of acquired points is named 'Point Cloud'. While the scanning with laser is happening, photos of the real world (with true colours RGB (Red Green Blue)) can be taken. These photos are used to assign to each point of the cloud the corresponding RGB value.

\subsection{Deformation assessment methods}

Deformation assessment methods consist of monitoring the integrity of structures to early detect potential damage and react appropriately in a timely fashion[7]. To carry out deformation analysis, there are three main approaches: 1) Point-to-Point, 2) Point-toSurface and 3) Surface-to-Surface.

Point-to-Point analysis:

If the deformation analysis is performed on two triplets of coordinates that represent the same position, but in two different dates, a simple comparison between the two triplets of coordinates gives the corresponding deformation. However, if we have two point clouds representing a scene in two different periods, it is uncertain that the exact same point appears in the two clouds for many reasons (different density, different scanning points...). To overcome this problem, a range image is generated from each point cloud based on the same point of perspective [8]. The value of each pixel of the range image corresponds to the distance to the scanning station. Then, pixel values from the two generated range images are subtracted to assess the deformation.

\section{Point-to-Surface analysis:}

In the Point-to-Surface analysis, a point cloud is compared to a reference surface. This surface may be an existing 3D model of the object of interest, or it may be generated from another point cloud by using specific techniques such as tessellation or surface fitting. The deformation corresponds to the shortest distance that is calculated according the normal of the reference surface [9].

Surface-to-Surface analysis:

Surface-to-Surface analysis allows comparing between two surfaces, that may come from existing 3D models or that are generated from two different point clouds. This 
type of analysis requires defining a mathematical parameterization for planar patches of each surface[10]. Then, the plan parameters and their covariance matrices are used to perform Surface-to-Surface analysis for deformation assessment [11].

\subsection{Discussion}

The use of total station may be considered as a conventional method since it allows using a set of discrete points across the structure (very limited number of points) in the process of deformation assessment. When using a total station in deformation monitoring, the $3 \mathrm{D}$ coordinates of specific points on the structure may be measured and compared to their coordinates on a model that represents the ideal condition of the building. In addition, it is possible to make different measurements of the same point at a regular interval of time, so one can deduce information about possible deformation on the structure over the time.

On the contrary, Close Range Photogrammetry (CRP) and Terrestrial LiDAR offer the possibility to acquire a very dense $3 \mathrm{D}$ point cloud that may be used in the process of deformation assessment. This 3D point cloud may be compared to an existing model representing the ideal condition of a structure. Also, if we have a set of data corresponding to two different dates, a deformation analysis may be carried out through a comparison between the two point clouds. CRP is very efficient to record the texture of elements and to automate extraction of features in the structure. However, Terrestrial LiDAR is much more appropriate for complex structures where the use of CRP may require complex processing [12]. Terrestrial LiDAR can capture the full surface of an object, even cracks and tiny deformations and map them up to millimeters of accuracy. In addition, the texture is acquired through the photos that are simultaneously taken during the scanning process.

\section{The combination of BIM and Terrestrial LiDAR for documentation and deformation assessment}

The use of a Terrestrial LiDAR allows a digital archiving of the as-built condition of a building. Indeed, Terrestrial LiDAR provides a very dense and accurate point cloud that captures the current condition of this building. Then, based on the acquired point cloud, a 3D model could be generated. This 3D model may represent either the as-built condition or the ideal condition of such a building.

In this section, we will introduce the concept of Building Information Modelling (BIM) and how it can be used to assess the deformation of historical buildings.

\subsection{The importance of BIM in cultural heritage}

Building Information Models (BIM) differ from conventional 3D models because BIM are based on knowledge-rich parametric building elements[13]. These parametric objects, which represent the components of the building, are assembled together to automate the generation of building information and to facilitate the creation of rich building models[13]. The parametric objects are defined according to a systemic approach where each object is characterized by its descriptive data and its relationship with other components of the building [14]. The concept of BIM is widely used in the field of cultural heritage preservation [15 - 18]. In [19] a plug-in prototype library, named Historical BIM (HBIM), for parametric objects used to model historical monuments has been developed. HBIM offers interactive parametric objects that represent architectural elements, and it was used to model historical monuments from TLS point cloud [14]. The BIM provides a powerful approach to automate the documentation of cultural heritage thanks to its parametric modelling process. It allows generating highly sophisticated 3D models and offer advanced visualization tools.

\subsection{The integration of LiDAR and BIM for deformation assessment}

The proposed approach for deformation assessment consist of integrating Terrestrial LiDAR with Building Information modelling. First, we proceed to the scanning of the exterior facades, then the interior components of the building. It is necessary that all scanning stations must be referenced according the same reference system, so we can register all stations in the same point cloud. After cleaning the resulting point cloud, the next step is to create a 3D BIM from this cloud. For this purpose, it is possible to use some commercial software such as Autodesk' Revit and Graphisoft's ArchiCAD [20]. This BIM must represent the ideal condition of the building because it will be compared to the point cloud corresponding to the as-built condition. Once the BIM is completed, the point cloud will be segmented according to the components of the BIM against which deformations will be assessed. Indeed, each segment of the point cloud is compared to the corresponding BIM component through a Point-to-Surface deformation analysis (see section 2.2). The result of such a comparison will provide a useful information about the current condition of the assessed part of the building, and hence if any restoration is needed. 


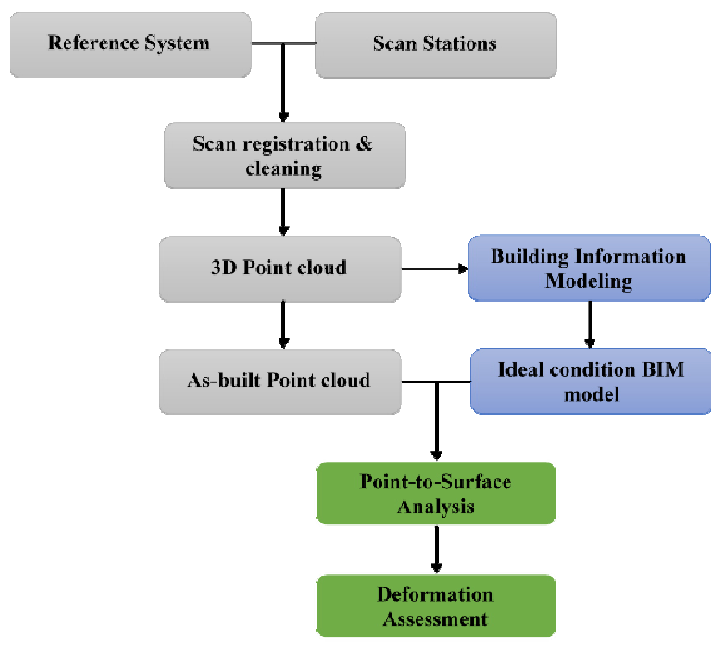

Fig. 1. A typical workflow of the proposed approach.

Figure 1 shows a typical workflow of the proposed approach for documentation and deformation assessment of historical buildings.

\section{Case Study: 'Robat Banajah'}

'Robat Banajah' is one of the most important historical buildings in Jeddah old city in the Kingdom of Saudi Arabia. This building was built to serve pilgrims that want to perform the fifth pillar of Islam. Then, it was endowed as a charity housing for widows and disabled. This 'Robat' contains 12 rooms on the ground level with a total area of around $430 \mathrm{~m}^{2}$. The architectural characteristics of the building are very simple with some plaster decorations on the main entrance (Figure 2).

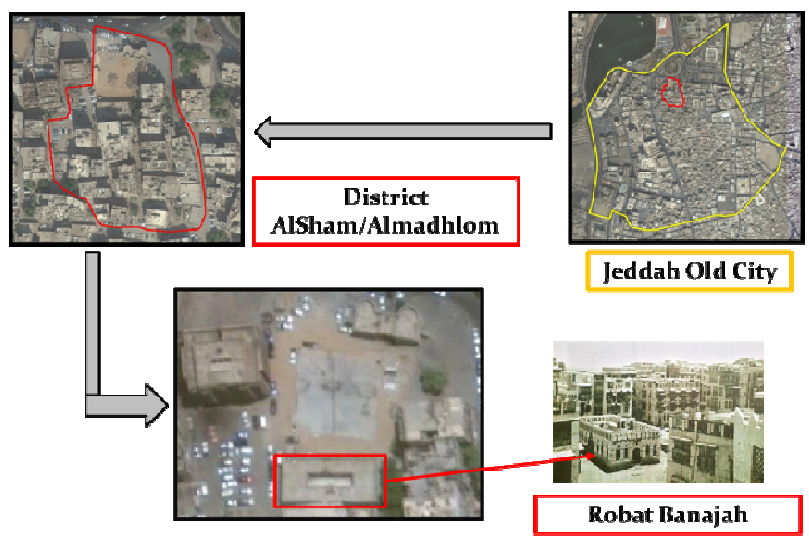

Fig. 2. Robat Banajah.

The acquisition of the current condition of the 'Robat' was performed by the 3D Laser Scanner Leica ScanStation C10. This instrument has a field of view of $360^{\circ}$ in the horizontal direction and $270^{\circ}$ in the vertical direction. It has a position precision of $6 \mathrm{~mm}$ (till $50 \mathrm{~m}$ range) and a maximum range of $300 \mathrm{~m}$.

Before proceeding to the scanning, it is necessary to define GCPs (Ground Control Points) according to the local reference system (Ain el Abd UTM 37 North). Leica GNSS Viva GS15 receivers (with a post processing horizontal precision $3 \mathrm{~mm}+0.5 \mathrm{ppm}$ ) are used in addition to a Leica Total Station TCR 1201+ (0.3 mgon,
$1 \mathrm{~mm}+1.5 \mathrm{ppm}$ with reflector). The orthometric height values of those GCPs are obtained through a geometric levelling by using a Leica Runner 24 level $(2.0 \mathrm{~mm}$ per $\mathrm{km}$ double-run levelling). The GCPs are used to have a georeferenced 3D point cloud that contains data about the exterior and the interior of the building.

The following figure shows the various surveying instruments that are used in the acquisition of the current condition of the historical building.
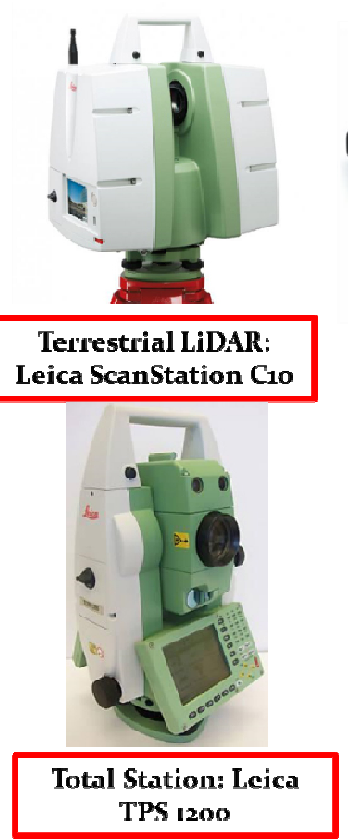

Leica ScanStation C1o

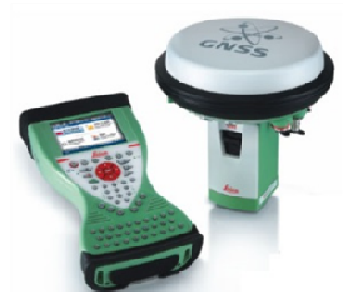

GNSS Receivers: Leica Viva
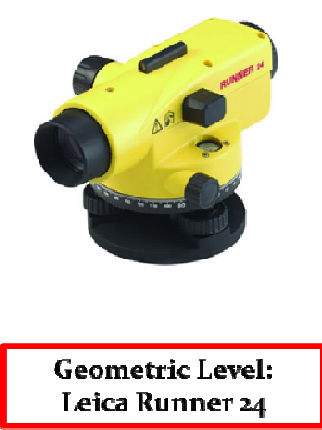

Fig. 3. Surveying instruments used in the acquisition of the current condition of the historical building.

The workflow of the scanning process consists of setting up the laser scanner on a traverse point with known coordinates and to have a known backward azimuth (backward traverse point with known coordinates). Then, we start acquiring forward traverse point with unknown coordinates, in addition to black and white targets that are used in the point cloud registration process. After that, a fully 3D point cloud acquisition is performed from the occupied point by the laser scanner (Figure 4).

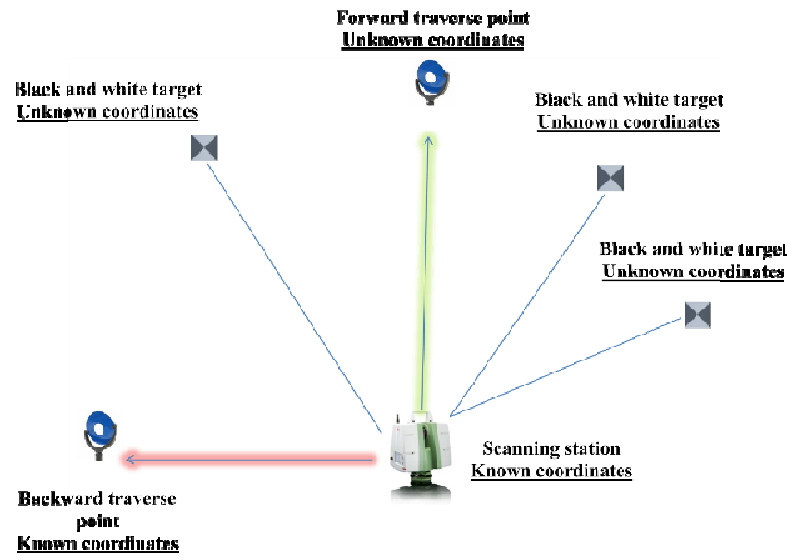

Fig. 4. The Laser scanning workflow. 
All scans that come from different scanning stations will be grouped together thanks to common targets. This process is called 'Registration'. The result is a unified point cloud, where each point has 3D coordinates according to the local reference system (Ain el Abd UTM 37 North). The following figure shows the 3D registered point cloud of 'Robat Banajah'.

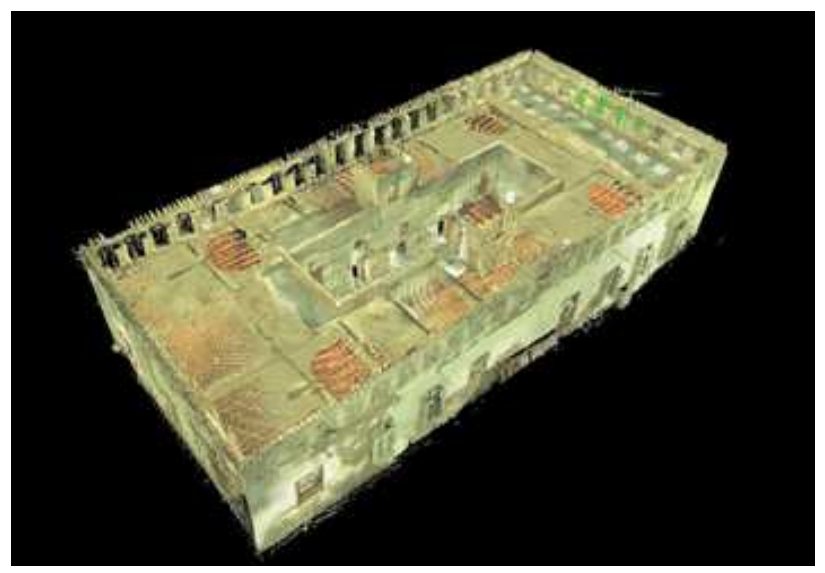

Fig. 5. Registered 3D point cloud of 'Robat Banajah'.

The obtained point cloud is then used to produce a 3D BIM model that represents the ideal condition of the building (Figure 6). This BIM must include the main parts of the building that should be assessed such as walls, ceilings, roofs, etc.

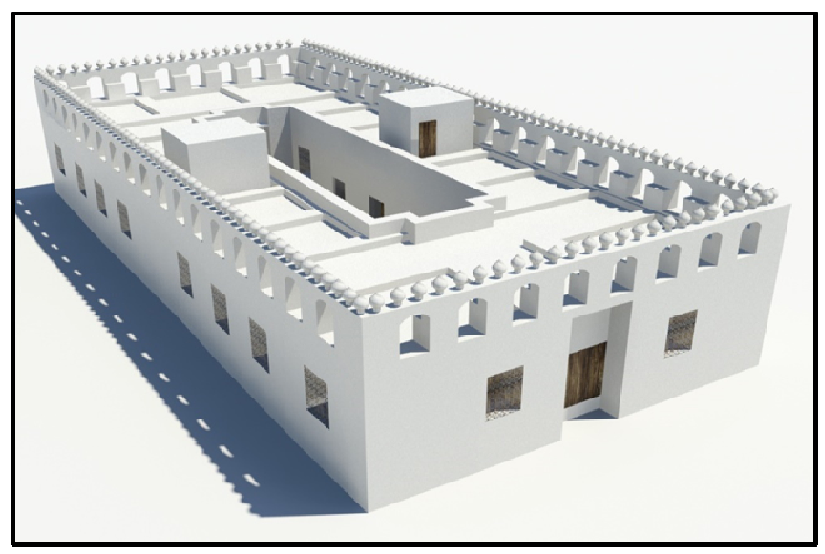

Fig. 6. The 3D BIM model of 'Robat Banajah'.

Before proceeding to deformation analysis, we need to segment the point cloud into parts that will be compared to the corresponding parts of the BIM. In this case study, we are interested in walls and ceilings. So, the first step is to isolate the room (or the set of rooms) to be assessed. Then, the ideal ceiling that comes from the BIM model is used in a Point-to-Surface deformation analysis. This analysis gives a map of differences between the used point cloud and the ideal plan representing the ceiling. The following figure shows the result of deformation analysis on one room of 'Robat Banajah' which has the most deformation values.
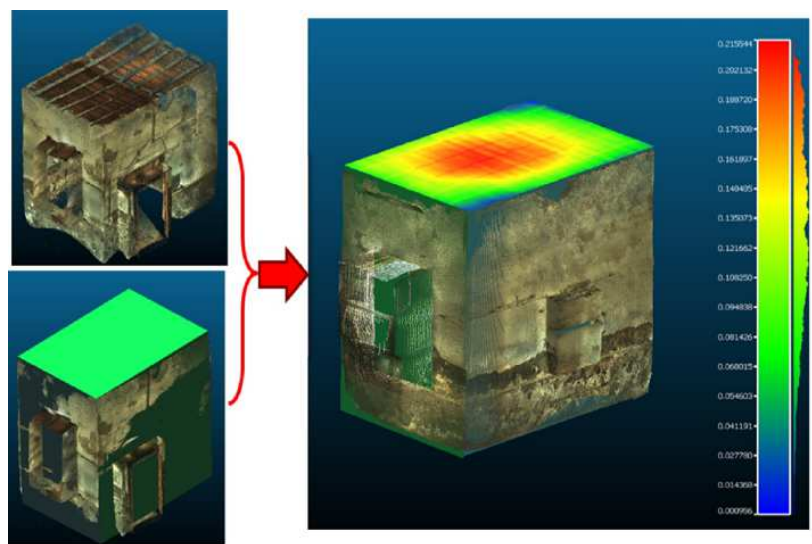

Fig. 7. Deformation analysis of a room ceiling.

The deformation analysis of this room shows that the maximum ceiling deflection is about $22 \mathrm{~cm}$. Also, more than $50 \%$ of the deflection measurements were more than $10 \mathrm{~cm}$ which may require restoration and maintenance works for that ceiling.

It is also possible to make deformation analysis on walls as shown in the following figure.

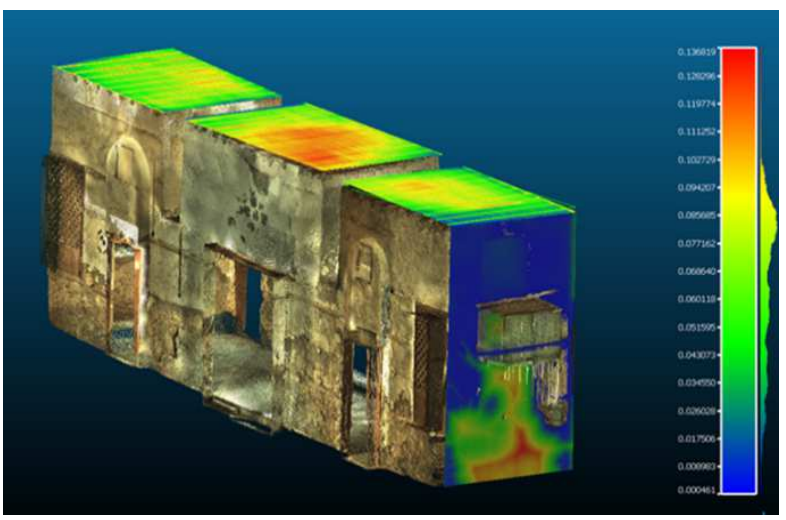

Fig. 8. Deformation analysis of ceilings and walls.

\section{Conclusion}

The aim of this study is to propose an approach for heritage documentation and deformation assessment based on the combination of Terrestrial LiDAR and Building Information Modeling. Terrestrial LiDAR is a very interesting method to provide digital archiving of cultural heritage as a $3 \mathrm{D}$ point cloud. This point cloud is characterized by its very high density. In addition, each point of the cloud has very accurate $3 \mathrm{D}$ coordinates according to a specific reference system. Also, the point cloud capture information about the texture of the building thanks to realistic photos that are taken during the scanning process.

One of the most important use of Terrestrial LiDAR point cloud is the creation of $3 \mathrm{D}$ Building Information models. These 3D models can be used in different applications such as archaeology, history and culture. In this paper, we have highlighted the importance of BIM in the field of deformation assessment. The case study of 'Robat Banajah' shows how a Point-to-Surface analysis 
can be performed to assess the current condition of an historical building. Results show that some parts of the building have a distortion of about $22 \mathrm{~cm}$, which means that further investigation must be carried out by specialists to decide if some restoration and maintenance interventions are required.

\section{References}

1. Roca, P., Cervera, M., \&Gariup, G. (2010). Structural analysis of masonry historical constructions. Classical and advanced approaches. Archives of Computational Methods in Engineering, 17(3), 299-325.

2. Callegari, F. (2003). Sustainable development prospects for Italian coastal cultural heritage: a Ligurian case study. Journal of Cultural Heritage, 4(1), 49-56.

3. Zulkepli Majid, Halim Setan, and Albert K. Chong. (2009). Accuracy assessments of point cloud 3D registration method for high accuracy craniofacial mapping. Geoinformation Science Journal, 9 (2). pp. 36-44.

4. Grussenmeyer, P., Hanke, K., Streilein, A. Architectural photogrammetry. in Digital Photogrammetry, edited by M. KASSER and Y. EGELS, Taylor \& Francis (2002), pp. 300-339.

5. Remondino, F., \& El $\square$ Hakim, S. (2006). Image $\square$ based 3D modelling: a review. The Photogrammetric Record, 21(115), 269-291.

6. Wehr, A. (2008). LIDAR: Airborne and terrestrial sensors. In: Z. Li, J. Chen and E. Baltsavias (red.), Advances in Photogrammetry, Remote Sensing and Spatial Information Sciences: 2008 ISPRS Congress book.

7. Armesto, J., Arias, P., Roca, J., \& Lorenzo, H. (2008). Monitoring and assessing structural damage in historic buildings. The Photogrammetric Record, 23(121), 36-50.

8. Douillard, B., Underwood, J., Kuntz, N., Vlaskine, V., Quadros, A., Morton, P., \&Frenkel, A. (2011, May). On the segmentation of 3D LIDAR point clouds. In Robotics and Automation (ICRA), 2011 IEEE International Conference on (pp. 2798-2805). IEEE.

9. Barnhart, T. B., \& Crosby, B. T. (2013). Comparing two methods of surface change detection on an evolving thermokarst using high-temporal-frequency terrestrial laser scanning, Selawik River, Alaska. Remote Sensing, 5(6), 2813-2837.

10. Lindenbergh, R., \& Pfeifer, N. (2005, October). A statistical deformation analysis of two epochs of terrestrial laser data of a lock. In Proceedings of the 7th Conference on Optical (pp. 61-70).

11. Mukupa, W., Roberts, G. W., Hancock, C. M., \& AlManasir, K. (2017). A review of the use of terrestrial laser scanning application for change detection and deformation monitoring of structures. Survey Review, 49(353), 99-116.

12. Soni, A., Robson, S., \&Gleeson, B. (2015). Structural monitoring for the rail industry using conventional survey, laser scanning and photogrammetry. Applied Geomatics, 7(2), 123-138.

13. Lee, G., Sacks, R., \& Eastman, C. M. (2006). Specifying parametric building object behavior (BOB) for a building information modeling system. Automation in construction, 15(6), 758-776.

14. Murphy, M., McGovern, E., \& Pavia, S. (2013). Historic Building Information Modelling-Adding intelligence to laser and image based surveys of European classical architecture. ISPRS journal of photogrammetry and remote sensing,76, 89-102.

15. Li, Y., Dong, K., \& Li, G. F. (2014, November). The Application of BIM in the Restoration of Historical Buildings. In Applied Mechanics and Materials (Vol. 638, pp. 1627-1635).

16. Baik, A., Alitany, A., Boehm, J., \& Robson, S. (2014). Jeddah Historical Building Information Modelling. ISPRS Annals of Photogrammetry, Remote Sensing and Spatial Information Sciences, 1, 41-47.

17. Di Mascio, D., Pauwels, P., \& De Meyer, R. (2013). Improving the knowledge and management of the historical built environment with BIM and ontologies: the case study of the Book Tower. In 13th International Conference on Construction Applications of Virtual Reality (pp. 427-436).

18. Bogdanova, G. T., \&Atanasova, V. (2013). Digitisation and Presentation of Historical Materials in a Virtual Exhibition 'The Image of India in Bulgaria: from the late 19th to the late 20th Cen. Digital Presentation and Preservation of Cultural and Scientific Heritage, (III), 212-218.

19. Dore, C., \& Murphy, M. (2012, September). Integration of Historic Building Information Modeling (HBIM) and 3D GIS for recording and managing cultural heritage sites. In Virtual Systems and Multimedia (VSMM), 2012 18th International Conference on (pp. 369-376). IEEE.

20. Brilakis, I., Lourakis, M., Sacks, R., Savarese, S., Christodoulou, S., Teizer, J., \&Makhmalbaf, A. (2010). Toward automated generation of parametric BIMs based on hybrid video and laser scanning data. Advanced Engineering Informatics, 24(4), 456465 . 